\section{Un crisol de experiencias: la extensión universitaria en dos regiones de México}

León Tomás Ejea Mendoza

Universidad Autónoma Metropolitana-

Azcapotzalco, México.

tomas.ejea@gmail.com

(iD) orcid.org/0000-000265976894
Ahtziri Molina Roldán

Universidad Veracruzana, México.

ahtziri@gmail.com

(iD) orcid.org/0000-0001-6722-4787
Ambiente y extensión universitaria /

Perspectivas

다)(이잉
RECEPCIÓN: 30/06/21

ACEPTACIÓN FINAL: 28/09/21

\section{Resumen}

En este artículo presentamos los modos en que los programas de extensión universitaria de las Instituciones de Educación Superior de dos regiones geográficas de México se relacionan con su entorno respecto de la cuestión ambiental: la región del Estado de Chiapas y la región denominada Riviera Maya. Se puede decir que Chiapas, con su fuerte composición de población indígena comunitarista, presenta una tendencia a un "enfoque ecologista", y que en la Riviera Maya, con su carácter de centro turístico internacional de lujo, predomina un "enfoque economicista". Con ello, tenemos que las intervenciones y las relaciones con el entorno de las Instituciones de Educación Superior en México poseen una gran diversidad de opciones, lo cual permite afirmar que es un verdadero crisol de experiencias.

Palabras clave: educación superior en México extensión universitaria; territorio; universidad.
A melting pot of experiences: university extension programs in two regions of Mexico

\section{Abstract}

In this article, we present how the university extension programs of the Higher Education Institutions of two geographical regions of Mexico are related to their surroundings on environmental issues: the Region of the State of Chiapas and the region called the Riviera Maya. It can be said that in Chiapas, with its strong composition of the communitarian indigenous population, there is a tendency towards an ecological approach. For its part, the Riviera Maya, as a tourist centre of international luxury, tends towards the economistic approach. In this sense, we show that the forms of intervention and relationship of higher education institutions in Mexico with their surroundings present a great diversity of options that allow us to assert that it is a true melting pot of experiences.

Keywords: higher education in Mexico; university extension; territory; university.
Uma mistura de experiências: extensão universitária em duas regiões do México

\section{Resumo}

Neste artigo, apresentamos as formas como os programas de extensão universitária das instituições de Ensino Superior nas regiões geográficas do México se relacionam com seu meio ambiente em questões ambientais: a Região do Estado de Chiapas e a Região denominada Riviera Maya. Pode-se dizer que em Chiapas, com sua forte composição de população indígena comunitária, haveria uma tendência para uma abordagem ecológica e, por sua vez, na Riviera Maya, com seu carácter como centro turístico de luxo internacional, teria uma tendência da abordagem economicista. Nesse sentido, pode-se dizer que as formas de intervenção e ralação com o meio ambiente das instituições de ensino superior no México, apresentam uma grande diversidade de opções que nos permitem afirmar que se trata de uma verdadeira mistura de experiências.

Palavras-chave: ensino superior no México; extensão universitária; território; universidade.

Para citación de este artículo: Ejea Mendoza, L. T. y Molina Roldán, A. (2021). Un crisol de experiencias: la extensión universitaria en dos regiones de México. +E: Revista de Extensión Universitaria, 11(15), e0006. doi: 10.14409/extension.2021.15.Jul-Dic.e0006 


\section{Introducción}

En la medida en que el cuidado ambiental y el desarrollo sustentable se han tornado dos de las problemáticas más importantes a nivel global, las instituciones de educación terciaria también se han ido involucrando en la tarea de buscar soluciones para una mejor convivencia entre el ser humano y la naturaleza. Los planos de participación que tiene la universidad en esta problemática están dados en la formación de profesionistas no solamente abocados a las áreas específicas de esta materia, sino, en general, comprometidos con las necesidades ambientales y el desarrollo sustentable. A la vez, la universidad tiene un papel social central en la generación de conocimiento a través de la investigación acerca de las necesidades económicas, técnicas y sociales para lograrlo, pero también realiza actividades de extensión universitaria y difusión de la cultura que ponen en el centro de la atención la necesidad de realizar proyectos de enlace comunitario y de vinculación con los sectores sociales, gubernamentales y privados, que favorezcan el desarrollo económico, social y cultural, con un sentido sustentable (Bohne, 2019; Moneva y Vallespín, 2012; De Vries y Rivera, 2017; Yanez y Zavarce, 2011).

A este respecto, se puede decir que las formas de intervención y de relación con el entorno de las Instituciones de Educación Superior (IES) públicas en México son diversas, lo cual nos lleva a afirmar que es un verdadero crisol de experiencias.

Bajo estas premisas, en el presente artículo abordamos los modos en que se relacionan con su entorno en cuanto a la cuestión ambiental las IES públicas la región del Estado de Chiapas y la denominada Riviera Maya. Ambas son parte de la zona climática cálida tropical de México, tienen una fuerte composición de población indígena y comparten, por su cercanía, problemáticas y formas culturales.

Lo que hemos encontrado, y es el motivo principal de este artículo, es que la forma de llevar a cabo la extensión universitaria en general, y en específico, en materia de medio ambiente y sustentabilidad económica, difiere en cada región. Se podría decir, siguiendo los planteamientos de Bustillo y Martínez (2008), que en Chiapas habría una tendencia al enfoque ecologista y que en la Riviera Maya, con su carácter de centro turístico internacional de lujo y de alta envergadura, utilizando los términos de los mismos autores, habría una tendencia a un enfoque economicista (Bustillo y Martínez, 2008).

Cabe mencionar que este artículo es un producto de la investigación denominada "Modelos de tercera función sustantiva universitaria y su relación con el entorno", ' que se desarrolló desde agosto de 2015 a enero de 2019, y representa el primer acercamiento sistematizado y global en el campo académico que pretende analizar los usos y las modalidades que las IES de carácter público de México llevan a cabo con respecto de la denominada Tercera Función Sustantiva Universitaria. Cabe decir que hemos denominado como la Tercera Función Sustantiva Universitaria a aquella que engloba las actividades universitarias que, no siendo propiamente ni la docencia ni la investigación, se enfocan en lo que se llama extensión universitaria, difusión cultural, vinculación social.

El objetivo de esta investigación fue analizar las modalidades y los usos que cada institución de educación terciaria da a esta función —extensión universitaria, difusión cultural

1) Otro producto que se generó a partir de esta investigación es el libro Entre la comunidad y el mercado, los ámbitos y usos de la tercera función sustantiva universitaria en México, de los autores Ahtziri Molina Roldán y Tomás Ejea Mendoza (2019). 
y vinculación social一, es decir, cómo se vincula con su entorno. Para lograr el objetivo se realizó un trabajo de campo en 36 IES públicas de México divididas en seis regiones geográficas del país.

Figura 1. Mapa de México con las seis regiones estudiadas

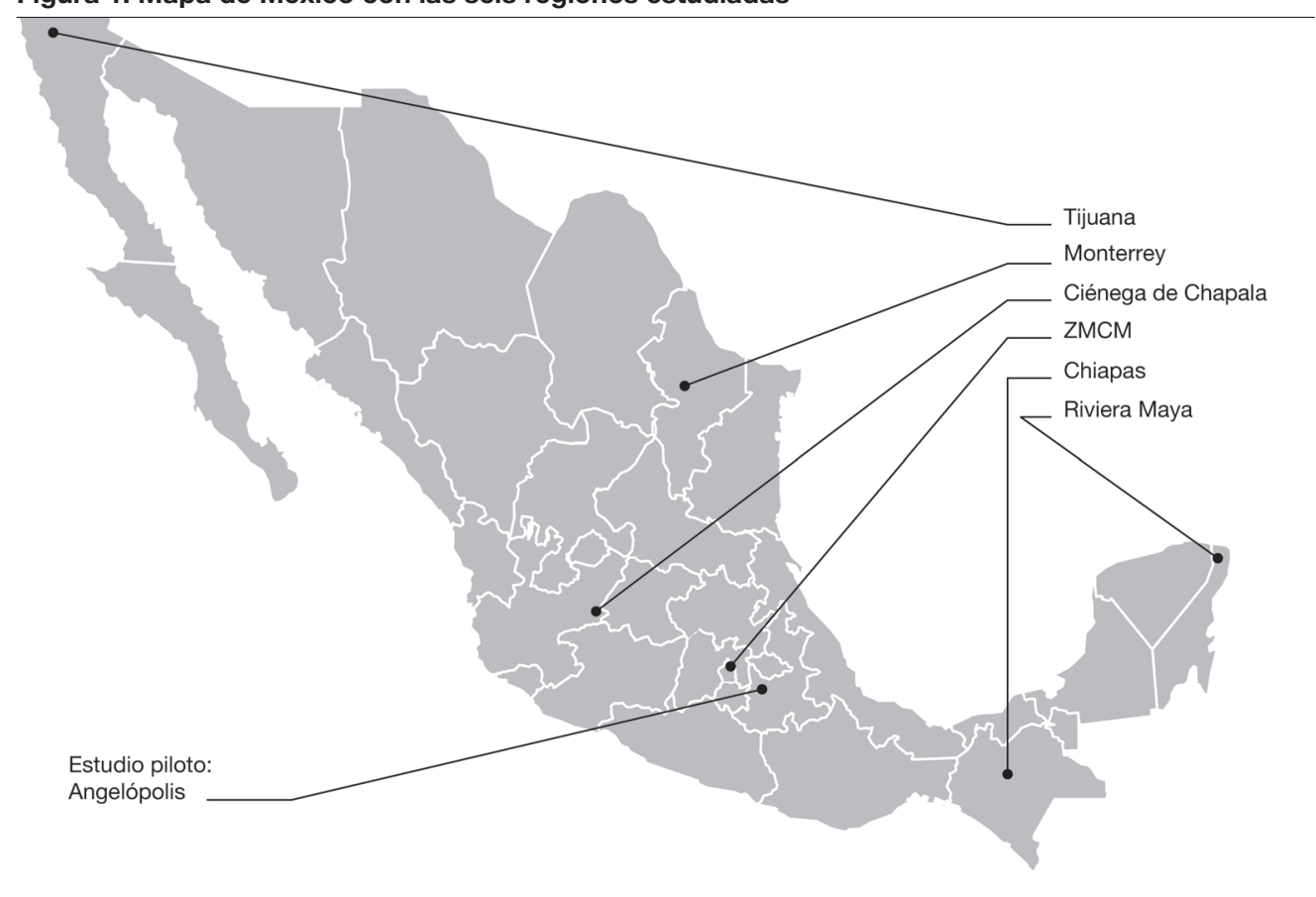

Fuente: Itzel Sainz.

Como se observa en la Figura 1, se establecieron seis regiones del país con características específicas para tener una representatividad de la diversidad económica, social y cultural de toda la nación. ${ }^{2}$ En cada región se seleccionaron seis IES públicas, lo cual hace un total de 36 instituciones estudiadas en esta investigación.

A partir de ello, en este artículo se presentan únicamente los resultados puntuales de dos IES de dos de esas regiones, puesto que presentar el total de instituciones de cada región resultaría realmente muy extenso. Tomamos las regiones de Chiapas y la Riviera Maya puesto que tienen en común tres aspectos: primero, las dos se encuentran geográficamente muy cercanas en el sureste del país (tal como se puede ver en el mapa respectivo). Segundo, ambas tienen un fuerte componente de población indígena, que habla lenguas mayenses,

2) Las seis regiones estudiadas son: región de la ciudad de Tijuana en el Estado de Baja California, como una ciudad binacional con una economía basada en la industria y la migración con el Estado de California de Estados Unidos. Región Metropolitana de Monterrey en el Estado de Nuevo León, por su carácter industrial. Región Agrícola de La Ciénega del Lago de Chapala que involucra a los Estados de Jalisco y Michoacán y tiene una identidad fundamentalmente agrícola. Región de la ciudad de México, como capital del país y por su alta concentración de población y oferta educativa a nivel superior. Región del Estado de Chiapas como zona de alta marginación y gran presencia de población indígena. Región de la Riviera Maya en el Estado de Quintana Roo como zona de turismo internacional y alta presencia de población indígena. 
composición que es propia del sureste del país. Y tercero, al igual que todo el país, pertenecen a un sistema de educación superior que en muchos aspectos puede considerarse como muy diverso en términos de la especificidad de las instituciones que lo componen pero que constituye una unidad que rige de igual manera para ambas regiones estudiadas. Sin embargo, si bien ambas se encuentran en zonas indígenas y los respectivos sistemas educativos son muy recientes, la forma de entendimiento, la aplicación y la viabilidad de las políticas centrales toman rumbos distintos debido a las características propias de la población, del desarrollo económico, social y territorial que cada una ha seguido. Esto es, se debe tener en cuenta que la región de Chiapas está conformada como una sociedad tradicional de larga data que, desde los tiempos de la Colonia, siglo XVI, ya era una sociedad constituida como tal. Por el contrario, la Riviera Maya es un desarrollo turístico creado hace apenas cinco décadas, con visos de lograr un centro de servicios moderno y funcional que fue construido muy cerca pero independiente de los asentamientos indígenas de la región. Es precisamente esta distinción territorial lo que hace que las IES establezcan distintas formas de llevar a cabo sus actividades y de relacionarse con el entorno.

Este artículo se estructura en cuatro apartados: en el primero se hace una breve revisión de algunos términos que resultan necesarios para dar un marco conceptual que establezca la lógica del artículo. Los términos son territorio, extensión universitaria, tercera función sustantiva universitaria y cuidado ambiental.

En el segundo apartado se analiza el caso de la región del Estado de Chiapas y se reflexiona acerca de los elementos que nos llevan a entender su particularidad en cuanto a su carácter indígena y de tradición comunitaria pero con un gran peso de los cacicazgos políticos y económicos de grupos mestizos, a la vez que se presenta su problemática conflictiva actual con respecto a la rebelión y los casos contestatarios al orden establecido puntualmente relacionados con el movimiento del Ejército Zapatista de Liberación Nacional (EZLN).

En el tercer apartado se lleva a cabo una tarea similar para el caso de la Rivera Maya. Igualmente, se comentan los aspectos reflexivos, de corte económico, social y cultural, que permiten entender a esta región como una zona de muy reciente creación pero con un rápido desarrollo económico y poblacional, con una lógica dirigida al turismo de lujo de corte internacional que ha dado pie a una industria fundamentalmente hotelera y de servicios vinculados a lo turístico. Con ello, hacemos ver que las IES de esta región se han dedicado a concebir la relación con su entorno y con el medio ambiente buscando en especial la generación de fuerza de trabajo y de condiciones para el desarrollo del mercado del turismo y de los sectores económicos lindantes.

Posteriormente, pasamos a desarrollar el tipo de extensión universitaria y difusión cultural que se realiza en las seis instituciones estudiadas en esta región del país. Y para finalizar presentamos un breve apartado en el que exponemos algunas ideas a manera de conclusiones.

\section{Algunos elementos conceptuales: territorio, extensión universitaria y cuidado ambiental}

En lo que respecta a la regionalización que hemos hecho, partimos del principio de que la geografía no es únicamente una ciencia descriptiva sino, tal como dice Gasca: "La agenda de temas de la geografía regional es sumamente variada... se han centrado en el estudio 
de las instituciones y los agentes de cambio, así como la espacialidad de los procesos materiales, cultural, discursivos y simbólicos" (2009, p. 32). En este sentido, creemos también que las regiones no son solo un espacio "natural" sino que son lugares de lucha y de comunicación donde los intereses económico-políticos y las interrelaciones simbólicas se conjugan para establecer lugares de dominio y de hegemonía. La territorialización se vuelve fundamental como variable para entender las tensiones y las relaciones sociales que se viven tanto al exterior de la universidad como al interior de la misma. Como señala Erreguerena:

"Resulta clave que la lectura crítica acerca del territorio la dirijamos también hacia la propia universidad, al equipo y al proyecto de extensión, aun en la etapa de formulación. Ello en el sentido de interrogarnos acerca de qué modelo de universidad, qué actores y estructuras de poder dentro del territorio, esta vez propio, universitario, el proyecto de extensión refuerza o problematiza". (2020, p. 10)

Por ello, consideramos que las dos regiones que hemos escogido para este artículo tienen procesos de territorialización distintos. Probablemente se pueda pensar que de manera "natural" tienen coincidencias tales como el clima, poblaciones con una larga tradición indígena, problemáticas agrarias, etc. Sin embargo, las formas de territorialización han sido muy distintas en las últimas décadas. Esto es, en la región de Chiapas se vive un proceso de desarrollo indígena apegado a una tradición ancestral, donde la resistencia de los pueblos frente a la forma de vida occidental o moderna ha sido una constante durante muchos siglos. En las últimas décadas, con el avance del desarrollo del capitalismo depredador, aunado a las políticas públicas tanto estatales como nacionales, se ha pretendido establecer un proceso de desarrollo extractivista y de desincorporación de la propiedad tradicional. Eso ha generado una importante resistencia entre estas comunidades, como la del EZLN, que comenzó en los años 90 del siglo pasado y desde entonces enarbola muchas de estas demandas aun hasta nuestros días.

Por otra parte, tenemos la región llamada la Riviera Maya, denominación reciente vinculada a la mercadotecnia turística de lo que es la costa del Caribe de la Península de Yucatán, que constituye un megaproyecto iniciado apenas en los años 70 del siglo pasado, ubicado en una zona del litoral en la que prácticamente no existía población alguna y que estaba rodeada de poblados de origen maya. Este megaproyecto significó un verdadero proceso de territorialización acelerado donde la cuestión del cuidado ambiental estuvo totalmente ausente y donde apenas hace unos años se han tomado medidas, entre ellas, las implementadas por las IES de la región.

Asimismo, partiendo de la premisa antes mencionada de que el espacio dentro de la universidad también es un territorio de diferentes visiones y en muchas ocasiones de proyectos opuestos (Arzeno, 2018 y Erreguerena, 2020), sucede lo mismo con la función de la extensión universitaria, que no puede ser definida unidireccionalmente. Consideramos que la extensión universitaria y, por tanto, la relación de la universidad con su entorno, están compuestas por múltiples elementos que representan diversas actividades directas o indirectas con respecto a la influencia y a la participación que tiene en el territorio "no universitario". Erreguerena lo plantea de esta manera:

"la categoría de territorio es usualmente utilizada para designar a un otro situado fuera de la universidad y rara vez es una herramienta para pensar y problematizar a la propia 
universidad como territorio. Territorio atravesado y construido por conflictos, alineamientos y territorializaciones diversas producto de la interacción de los claustros y distintos intereses del campo universitario". (2020, p. 11)

La influencia que tiene la universidad en su entorno no solo se da a través de proyectos definidos exclusivamente como de extensión universitaria, sino que de alguna manera la propia formación de profesionistas comprometidos, la investigación que se realiza en las IES o diferentes actividades de carácter cultural, también representan la relación que se establece con la sociedad. Es por ello que hemos optado por dotar al concepto de extensión universitaria de una tesitura nominal más amplia, que no se restringe a los proyectos definidos por cada institución como tales, y hemos propuesto ampliarlo utilizando la expresión "tercera función sustantiva universitaria". Con este término buscamos comprender también las tareas que hemos denominado "ámbitos", que comprenden la difusión cultural, el enlace comunitario y la vinculación con las instancias sociales, gubernamentales y privadas, entre otros. ${ }^{3}$

Tenemos muy en cuenta que, de acuerdo con Tommasino y Cano (2016), la extensión universitaria puede tener un carácter "difusionista-transferencista" o, por el contrario, un carácter de "extensión crítica". Por supuesto que abogamos por una extensión universitaria del segundo tipo o, para usar la terminología que nos interesa implementar: una tercera función sustantiva, que debe de tener una "vocación transformadora; y en ella, la importancia que otorga al vínculo educativo como elemento central en las relaciones de saber-poder que se establecen en el proceso de extensión" (Tommasino y Cano, 2016, p. 15). Sin embargo, el comprender esta dicotomía entre la extensión difusionista-transferencista y la extensión crítica, para designarlas sucintamente, y tener presente sus implicaciones económicas y políticas, no obsta para que en un ejercicio analítico ignoremos que, en general, dentro de la universidad latinoamericana se presentan ambas; algunas veces de manera concurrente; en algunas situaciones, una predomina claramente sobre otra; y en ocasiones se dan de modo alternado en el transcurso de la vida universitaria, pues esta coexistencia responde a las distintas visiones o proyectos, a menudo incluso antagónicos, que conviven en la institución universitaria.

Por ello, en este artículo buscamos mostrar cómo en las universidades de las dos regiones que hemos tomado como ejemplo se presentan al unísono y de forma compleja ambas visiones, pero con determinados matices que se explican a partir de las dos maneras en que se territorializó cada uno de esos espacios regionales. Por un lado, está el espacio de Chiapas, con una visión comunitarista que representa la presencia indígena siempre en resistencia, combinada con el intento institucional de los gobiernos central y estatal de mediatizar sus demandas mediante la fundación de instituciones educativas, más que para cumplir los acuerdos pactados, para quitarle presencia a la movilización zapatista. Por otro lado, está el proyecto gubernamental tanto del gobierno del Estado de Quintana Roo como del gobierno central federal de México por generar un polo de desarrollo moderno, turístico de gran calado de bienes, servicios e infraestructura, fuertemente ligado al capital internacional.

3) Para una explicación más amplia con respecto a la denominada Tercera Función Sustantiva universitaria se puede consultar nuestro libro ya mencionado: Entre la comunidad y el mercado: Los ámbitos y usos de la tercera función sustantiva universitaria en México. 
Con ello pensamos que resulta conducente plantear, junto con Bustillo y Martínez (2008), que las formas de relacionarse con el ambiente pueden estar dirigidas hacia un enfoque economista: "donde la naturaleza es vista como un instrumento en beneficio del hombre, para ser explotada... cuyo objetivo es apoderarse de la naturaleza para apoyar y beneficiar a la clase burguesa y por ende a su acumulación de bienes" $(2008$, p. 3) o a un enfoque de imperativo ecológico, en el que:

"se intuye que el valor intrínseco de los recursos naturales es reconocido independientemente del uso instrumental o el valor que los seres humanos les den... Donde el punto de atención principal es la conservación de los recursos y no el agotamiento de los mismos". (Bustillo y Martínez, 2008, p. 4).

Ahora bien, partiendo de que la universidad es un territorio en que los proyectos de extensión pueden convivir de manera a veces más o menos compleja, a continuación se presentan ambos casos regionales, Chiapas y la Riviera Maya, intentando establecer algunos rasgos particulares de cada uno. Para ello se utilizan como ejemplo dos universidades en cada región representativas de esta problemática, la Universidad Tecnológica de la Selva (UT de la Selva) y la Universidad Intercultural de Chiapas (UNICH) de la región de Chiapas, y la Universidad Tecnológica de Riviera Maya (UTRM) y la Universidad Intercultural Maya de Quintana Roo (UIMQROO) de la región de la Riviera Maya. ${ }^{4}$

\section{Región del Estado de Chiapas: la extensión universitaria como trabajo crítico con la comunidad y la sustentabilidad ambiental}

La geografía política nacional está compuesta por 32 Estados. En este apartado se caracteriza al Estado de Chiapas y se analizan dos de sus IES como casos de estudio: UT de la Selva y UNICH, las cuales son representativas de los modelos educativos y de extensión universitaria que adopta este Estado de acuerdo con su población y modos de organización con relación a la sustentabilidad.

\section{Características económicas, sociales y culturales}

Chiapas es el décimo Estado más grande de México y el más sureño, con 73311 kilómetros cuadrados y una población de 5217908 habitantes. (INEGI, 2016a). Cabe decir que tiene los peores índices del país en cuanto a pobreza se refiere; por ejemplo, la esperanza de vida es allí de 73 años, la más baja, y que está por debajo de la media mexicana $(75,2)$ (INEGI, 2016a). La desigualdad entre hombres y mujeres es notoria. Del conjunto de la población económicamente activa, el 71,4 \% es hombre y el 28,6, mujer.

La ocupación poblacional se distribuye de la siguiente forma: $45,9 \%$ en comercio y servicios, $39,3 \%$ en el rubro de agricultura, cría y explotación de animales, en el sector forestal, en la pesca y en la caza; un 14, $6 \%$ en la industria y la construcción; y un 0,2 \% de los

4) No está de más recordar que este texto es producto de una investigación de 36 IES distribuidas en 6 regiones de todo el país. En cada región se estudiaron 6 instituciones, tal fue el caso de Chiapas y de la Riviera Maya. Sin embargo, por cuestión de espacio aquí presentamos solamente dos IES de cada una de estas dos regiones. 
encuestados no especificó sector de ocupación (INEGI, 2016b). Como puede verse, el rubro del sector agropecuario es muy relevante.

En lo que refiere al Índice de Desarrollo Humano (IDH) para el Estado de Chiapas, el mismo es de 0,692 puntos, y en 2020 fue el más bajo del país (INEGI, 2016b).

Existen otros rasgos ineludibles para comprender al Estado de Chiapas y, en él, las características de las universidades y de las IES: las situaciones de conflicto en torno a la tierra entre organizaciones y comunidades indígenas y campesinas, grandes propietarios y empresas (y sus grupos armados), y el Estado (en particular sus instituciones policiales y militares). Todo esto sumado a la condición de Estado fronterizo. Ambas circunstancias - conflictos políticos y control de la inmigración centroamericana- hacen que se concentre en Chiapas una fuerte presencia militar, al punto de que hay territorios del Estado que se encuentran militarizados.

Las luchas campesinas e indígenas en Chiapas se remontan a la época de la conquista. En la época contemporánea, sin dudas, el punto más agudo de conflicto, y más notable por su trascendencia política, estuvo dado por la emergencia del EZLN el $1^{\circ}$ enero de 1994. Desde entonces, el Movimiento Zapatista y el desarrollo de su propuesta de autonomía se han convertido en una referencia central al aproximarse a la comprensión de este Estado. En efecto, la tensión entre autonomía comunitaria de los pueblos indígenas chiapanecos y el Estado federal se constituye en uno de los puntos principales para entender, por ejemplo, el tema de la educación en Chiapas.

Desde la irrupción del EZLN al presente, no sin oscilaciones, la respuesta del gobierno mexicano ha ido pasando de la represión militar como única respuesta a la combinación de la respuesta militar con otras de tipo asistencial (Harvey, 2003). De ahí la idea de fundar instituciones tales como la UT de la Selva y la UNICH.

\section{La extensión universitaria en dos IES en el Estado de Chiapas}

Las dos instituciones elegidas para la realización de este estudio son paradigmáticas, pues en el momento de su creación trajeron consigo modelos educativos de inicios del siglo actual, la búsqueda del desarrollo tecnológico en zonas más tradicionales, así como la interculturalidad. Aunque sus modelos pueden contrastarse, es importante mencionar que los dos contemplan como una necesidad la inclusión de los temas ambientales en sus programas curriculares y en sus actividades atinentes a la comunidad.

\section{UT de la Selva ${ }^{5}$}

Esta universidad nace de los Acuerdos de San Andrés Larráinzar firmados por el EZLN y el gobierno federal en febrero de 1996 debido a la demanda del Movimiento por una educación superior de calidad en las zonas indígenas de Chiapas. Inició sus actividades en 1997, y la matrícula estudiantil atendida en el ciclo 2016-2017 ascendió a 2789 alumnos. Forma parte del Subsistema de Universidades Tecnológicas (Secretaría de Gobierno de Chiapas, 1997, p. 3).

En su Misión se expresa que la UT de la Selva tiene como objetivo: "Brindar educación superior tecnológica de calidad, realizar investigación aplicada y difundir los valores de la 
cultura, para formar profesionistas íntegros, competitivos y emprendedores, comprometidos con el desarrollo del país y con el uso sustentable de los recursos naturales".

Asimismo, su Plan Institucional indica como línea de acción: "Consolidar la participación universitaria a través de proyectos de vinculación con la sociedad, principalmente con enfoque de sustentabilidad" (Secretaría de Gobierno de Chiapas, 1997, p. 4).

En el artículo 34 del Decreto de Creación se establece que se deben

"determinar los planes y programas que vincule a la Universidad Tecnológica de la Selva, con la sociedad, para actuar como agente de cambio y mejoría substancial de los niveles de ingreso y desarrollo, mediante la celebración de convenios de concertación, con grupos de campesinos, pequeños propietarios, industriales y comerciales, para desarrollar proyectos de integración de sociedades de producción, distribución, comercialización o consumo de bienes y servicios". (Secretaría de Gobierno de Chiapas, 1997, p. 4)

En lo que respecta al Servicio Social, el artículo 55 de la Ley de Educación para el Estado de Chiapas establece que se debe promover a los prestadores de servicio social en zonas marginadas y de bajo desarrollo:

"Artículo 55.- Los educandos que cursan el tipo superior, deberán prestar servicio social en los casos y términos que señalen las disposiciones reglamentarias, como requisito previo para obtener el título correspondiente. Todas las instituciones que proporcionen Educación Superior, observarán que los educandos cumplan con la prestación del servicio social acorde al perfil de su formación. Asimismo, promoverán que la prestación del servicio social de sus educandos se oriente preferentemente al apoyo de programas sociales dirigidos a zonas marginadas y de bajo desarrollo". (Poder Judicial, 2014, p. 64)

En el Plan Estatal de Desarrollo de Chiapas 2013-2018 se menciona como estrategia para la Educación superior: "Promover la participación de la población universitaria en la solución de problemas sociales y productivos, dentro y fuera de las universidades" (Gobierno del Estado de Chiapas, 2013, p. 114).

En tanto que en el Plan Institucional de la UT de la Selva se presenta como línea de acción: "Consolidar la participación universitaria a través de proyectos de vinculación con la sociedad, principalmente con enfoque de sustentabilidad" (UT. de la Selva, 2013, p. 40).

A nivel operativo, la UT Selva tiene un convenio firmado con la Comisión para el Desarrollo de los Pueblos Indígenas (CDI) en el que se despliegan acciones para atender la demanda de capacitación de algunas de las comunidades de los municipios aledaños, como Ocosingo, Sitala y Chilón, y de la región de la selva Lacandona. Los temas de la capacitación son sobre pequeños negocios, cuestiones sanitarias en bovinos, avícolas, porcinos, en hortalizas, entre otros temas para los grupos indígenas. Además, tiene una incubadora de negocios que ha buscado trabajar con los agricultores de la zona (UT de la Selva, 2016, p. 42).

A nivel operativo, esta universidad cuenta con Incubación de empresas, que es un Centro de Desarrollo especializado y el cual es importante mencionar puesto que no cobra por el servicio de incubación. El objetivo de la incubadora es disminuir el índice de fracaso para los pequeños comerciantes; se les asesora con herramientas administrativas, financieras, jurídicas, de imagen corporativa, protección de ideas y de marcas, entre otras. 
En lo referente a las estancias y estadías para los estudiantes, estas tienen una duración de cuatro meses de tiempo completo, aproximadamente de 6 a 8 horas diarias. Los alumnos pueden pasar estas estadías dentro de la universidad, y durante ese tiempo realizan una tesis, tesina o un proyecto, y tienen un tutor de la empresa. Los estudiantes salen de Ocosingo hacia otros Estados con mayor producción industrial, como Tabasco o Veracruz, para hacer sus estadías y estancias (UT de la Selva, 2016, p. 46)

El Departamento de Educación Continua se encarga de difundir el catálogo de servicios, que se divide en dos: Servicios Tecnológicos, que se dedica a la transferencia de tecnología del sector productivo, prototipos tecnológicos y cursos de capacitación. Y el Consejo de Vinculación y Pertinencia, a donde se invita a los sectores productivo, gubernamental y social para dar a conocer las carreras y servicios de la universidad, de lo cual participan empresarios, la Cámara de Comercio, la Secretaría de Educación y la Secretaría de Desarrollo Social, y se hacen convenios para prácticas, visitas, etcétera.

En cuanto a la vinculación con el sector productivo, su oferta es amplia, pues la mayoría de la vinculación es hacia empresas de la región y desde varios puntos del país a través de las prácticas así como del servicio de Incubadora Empresarial, el cual es gratuito para los pequeños empresarios de la región (UT de la Selva, 2016, p. 48).

$U N I C H^{6}$

La creación de esta universidad responde a la firma de los Acuerdos de San Andrés en febrero de 1996 entre el gobierno federal y el EZLN. Pertenece al Subsistema de Universidades Interculturales y contaba, en el ciclo 2015-2016, con 1876 alumnos. Cabe mencionar que las Universidades Interculturales se encuentran en regiones, municipios y zonas marginales con los menores IDH (UNICH, 2013, p. 16).

En el Decreto de Creación se expresa que las autoridades federales, estatales y municipales en materia de servicios de educación tienen la obligación de:

"Garantizar e incrementar los niveles de escolaridad, favoreciendo la educación bilingüe e intercultural, alfabetización, la conclusión de la educación básica, la capacitación productiva y la educación media superior y superior. Establecer un sistema de becas para los estudiantes indígenas en todos los niveles. Definir y desarrollar programas educativos de contendido regional que conozcan la herencia cultural de sus pueblos, de acuerdo con las leyes en la materia y en consulta con las comunidades indígenas, impulsar el respeto y conocimiento de las diversas culturas existentes en la Nación". (Poder Ejecutivo de Chiapas, Secretaría de Gobierno, 2004, p. 14)

Y en la misión de la UNICH se señala:

"La Universidad Intercultural de Chiapas es una Institución de Educación Superior, pública y descentralizada del Gobierno del Estado, que sobre la base del modelo educativo intercultural forma profesionistas de calidad, con valores, compromiso social y dominio de lenguas originarias de la entidad, a partir de la integración de conocimientos de los 
pueblos originarios y científicos para contribuir a la construcción de una sociedad con mejor calidad de vida. (UNICH, 2013, p. 16)

Sus principales programas docentes son: Desarrollo Sustentable, Turismo Alternativo, Comunicación Intercultural, Médico Cirujano y Derecho Intercultural. Los grupos indígenas atendidos en la UNICH son los de hablantes de lenguas tseltal, tsotsil, chol, zoque, tojolabal, zapoteco, mame, akateco, kanjobal, chuj, mixteco, mazateco y maya (CGEIB, 2015).

Esta universidad trabaja principalmente con las comunidades de la zona mediante un Proyecto Integrador que es obligatorio para todos los alumnos. Este debe tener impacto en la zona indígena, para lo cual se hace primero un diagnóstico para identificar las problemáticas de las zonas y después una visita física a la comunidad. Las comunidades que los alumnos eligen para el Proyecto Integrador pueden ser parte de servicio social, del proyecto terminal o del proyecto de tesis.

En el ámbito normativo, se establece en el Plan Estatal de Desarrollo Chiapas 2013-2018, en el Tema 2.3. Educación de calidad, Política pública 2.3.6. Educación Superior que: "Promover la participación de la población universitaria en la solución de problemas sociales y productivos, dentro y fuera de las universidades" (Gobierno del Estado de Chiapas, 2013, p. 114).

Al pasar al nivel operativo, el Departamento de Vinculación con la Comunidad y Servicio Social conecta a los jóvenes con los llamados Proyectos Integradores de Vinculación, con los que se busca tener un impacto en las comunidades concertadas. Por ejemplo, el vínculo desarrollado en la carrera de Medicina con la atención a dos municipios importantes San Andrés Larráinzar y Amatenango del Valle, proyectos hacia ciertas comunidades de alta marginación. Con los proyectos se pretende recopilar experiencias de la comunidad, de los jóvenes, de las asociaciones civiles con que se tiene convenio. También se realizó un convenio con la Universidad Juárez Autónoma de Tabasco, del que participaron quince jóvenes de la Licenciatura en Desarrollo Sustentable, quienes se certificaron y generaron proyectos y productos. Cada año se tiene alrededor de 80 prestadores de servicio social en la sede central.

Para este Departamento es relevante la vinculación con las lenguas originarias, pues es un requisito para todos los alumnos aprender una lengua originaria como las antes mencionadas, algunas de ellas en mucha menor cantidad, pero se busca que la vinculación pueda generar procesos, transformaciones, cambios de acuerdo con la vida comunitaria y tratar de recuperar los saberes locales (UNICH, 2013, p. 16).

Como se puede ver, para el caso de la región de Chiapas, muchas de las actividades de extensión están relacionadas con el trabajo comunitario. Es claro que la influencia de la movilización del EZLN, a más de 25 años de levantamiento armado, sigue siendo fundamental no solamente en la medida en que algunas instituciones se crearon a partir de su poder de negociación con el gobierno federal de aquel entonces, tales como la UNICH y la UT de la Selva, sino también por la presencia de programas de estudio y actividades de extensión que están ligados al concepto de comunidad indígena, como son el caso de "Vinculación permanente con la sociedad", "Salud Ambiental", "Proyecto Integrado con la comunidad" o "Ciudad Rural Sustentable"; así como los que están delineados para la conservación de las lenguas y tradiciones indígenas, como el de "Educación integral para todos", "Lenguas y culturas de Pueblos originarios" o "Festividades culturales", que juegan un papel relevante con respecto a la conservación y la preocupación por la cuestión ambiental, pues se puede 
decir que los esfuerzos van dirigidos a mantener un estado de equilibrio entre los seres humanos y la naturaleza que marca los valores culturales de las comunidades indígenas. Por otra parte, se observa otra vertiente en los esfuerzos por crear empresas a nivel local con la población o establecer contactos con los sectores productivos e industriales de la región, lo cual no está ligado necesariamente con lo comunitario y se ubica en el campo de las competencias comerciales y las capacidades individuales para hacerse de recursos, tales como los convenios de asesorías con servicios agroindustriales, de capacitación tecnológica, y los programas de incubadoras de empresas que cada vez se han vuelto más presentes en las IES del país. Con ello podemos comprobar que las acciones y programas tienen como foco ambos aspectos de la acción social: por una parte, el de ser un factor de desarrollo económico y comercial; y por otra, el de tener en mente el cuidado ambiental y de los patrones culturales de la población indígena.

\section{Región de la Riviera Maya: entre el turismo cosmopolita y el cuidado ambiental como desarrollo económico}

El Estado de Quintana Roo fue declarado como tal en 1974, lo cual lo convierte en uno de los de más reciente creación en el país. Su crecimiento poblacional comenzó en los años 70 y, por tanto, es una sociedad muy joven y con múltiples orígenes, pues, además de la población originaria, está compuesta por migrantes. La necesidad de proveer educación superior pública en todo el Estado es una demanda social reciente que ha sido provista de diversos modos. Tiene como una de sus principales patrimonios las ruinas de los pueblos mayas y su riqueza natural y, por ende, el manejo del medioambiente en la zona es primordial y en ello participan las IES.

\section{Características económicas, sociales y culturales}

La denominada Riviera Maya se encuentra en el ya mencionado Estado mexicano de Quintana Roo, que se localiza en el extremo oriental de México y abarca 50212 kilómetros cuadrados, lo que representa el 2,26 \% del territorio nacional.

Quintana Roo es considerado como uno de los lugares preferidos por turistas extranjeros y cuenta con el segundo aeropuerto más importante de la República. El sector de actividad que más aporta al PIB nacional, con 1, $6 \%$, es el turismo, esto es, servicios de alojamiento temporal y de preparación de alimentos y bebidas. Cuenta con una población de 1501562 habitantes, el 1,3\% del total del país. La edad media de la población es de 26 años. Posee el segundo lugar de tasa neta migratoria, $8,7 \%$ del total de habitantes del país. Además, tiene el primer lugar a nivel nacional en cuanto a proporción de residentes que no nació en su territorio. Su distribución poblacional es $88 \%$ urbana y $12 \%$ rural; de cada 100 personas, 17 son hablantes de una lengua indígena (INEGl, 2016c).

A nivel nacional, según el informe de Índice de Desarrollo Humano del Programa de las Naciones Unidas para el Desarrollo (PNUD), en el año 2020 Quintana Roo alcanzaba un indicador alto $(0,777)$; en comparación, hasta ese año la ciudad de México ocupaba el mayor nivel del país $(0,827)$ (PNUD, 2019).

El índice de marginación de Quintana Roo se considera medio; sus indicadores son menores que el promedio nacional, en tanto ocupa el vigésimo lugar a ese nivel. Solamente un 
indicador es mayor a la media, y responde a un $43,14 \%$ de viviendas con hacinamiento. Los municipios del Estado que presentan el índice medio de marginación son aquellos con mayor población indígena, a saber: José María Morelos, Felipe Carrillo Puerto y Lázaro Cárdenas.

A partir de la década de los 70, la historia del desarrollo económico del Estado de Quintana Roo estuvo ligada al turismo. La playa y el clima soleado eran los principales atractivos que buscaba el turismo internacional y, además de estos recursos, México ofrecía otros, como las zonas arqueológicas. El sector turístico permitía captar divisas, pero requería fuertes inversiones y una intensa mano de obra —situación que posibilitó vincular la ubicación de los nuevos centros turísticos con zonas marginadas del país, aspirando a generar empleos en las economías retrasadas-. Las zonas precisaban inversiones masivas en infraestructura turística y equipamiento urbano, lo que se complementaría con capitales privados (García ViIla, 1992). Debido a estos factores surgió la ciudad de Cancún como el primer Centro Turístico Integral planeado, y fue conocida también como "el sueño de los banqueros" (Pearce, 1991).

Desde 1974, la zona norte del Estado, con la ciudad de Cancún, comenzó su transformación. De ser un pequeño lugar de pescadores y cultivos de cocos, se convirtió en un polo de atracción del turismo internacional y uno de los centros turísticos de playa más importantes del país. Sus habitantes llegaron no solamente de Estados vecinos sino que se captaron trabajadores desde otros lugares más lejanos de México y del vecino Guatemala. Actualmente, los polos de atracción son las ciudades de Cancún, Playa del Carmen y Chetumal. ${ }^{7}$

Paralelamente a la industria del turismo, se han desarrollado zonas urbanizadas con población de clase media que tiene distintas ocupaciones en el mundo hotelero y en la atención de la población ya asentada en la zona. Asimismo, centros comerciales, ventas y servicios de distinta índole, planteles de educación pública y privada de todos los niveles, sectores de salud, de finanzas, y actividades culturales y artísticas de manera incipiente.

\section{La extensión universitaria en dos IES de la Riviera Maya}

Aquí se presentan dos casos que se ubican en distintas comunidades del Estado: una tradicional mayablante: la UIMQROO; y una de reciente creación en zona urbana, de crecimiento acelerado: la UTRM. Ambas ponen énfasis en el manejo del medio ambiente pero comprenden y tratan el tema de distintos modos.

\section{UIMQROO ${ }^{8}$}

Esta universidad se encuentra en el municipio de José María Morelos. Su año de fundación fue 2006, cuando surgió como parte del subsistema de Universidades Interculturales de México. Su oferta académica se compone de cinco licenciaturas, tres ingenierías y una maestría. En 2016 su comunidad estudiantil estaba integrada por 565 alumnos. A nivel normativo, dentro de sus objetivos se encuentra el reconocimiento del acervo lingüístico y cultural de los pueblos mayas.

7) Cabe destacar, por ejemplo, que la Riviera Maya es la región que por varios años ha tenido el primer lugar en recepción de turistas de cruceros en el mundo. https://qroo.gob.mx/sedetur/es-cozumel-quintana-roo-el-principal-puerto-de-cruceros-del-mundo

También se puede considerar el primer lugar turístico de "sol y playa" en América Latina con 21 millones de visitantes en 2019. https://qroo.gob.mx/sedetur/quintana-roo-primer-lugar-turistico-de-playa-en-mexico-y-america-latina 8) Para ver el caso de esta universidad con más amplitud se puede consultar el Reporte específico en Chiu (2017). 
El modelo pedagógico está basado en tres ejes articulados: la docencia, la investigación y la vinculación con las comunidades. Los tres ejes son considerados como experiencias fundamentales de aprendizaje y deben articularse entre sí en todo momento. Todo docente y todo alumno deben participar de los tres. Se privilegia la investigación sobre las lenguas y las culturas locales. La vinculación comunitaria, además de constituir una forma muy importante de aprender, significa un servicio a la comunidad que va creciendo en complejidad y significancia a medida que los alumnos van transitando por su carrera universitaria (Schmelkes, 2008, p. 9).

La misión de las Universidades Interculturales es promover la formación de profesionales comprometidos con el desarrollo económico, social y cultural, particularmente, de los pueblos indígenas del país y del mundo circundante; revalorar los conocimientos de los pueblos indígenas y propiciar un proceso de síntesis con los avances del conocimiento científico; fomentar la difusión de los valores propios de las comunidades, así como abrir espacios para promover la revitalización, desarrollo y consolidación de lenguas y culturas originarias (Secretaría de Educación Pública, 2017).

Con respecto al plano normativo, los objetivos XI y XII del Decreto de Creación de la UIMQROO hacen señalamientos sobre esta vertiente de la tercera función sustantiva:

"Objetivo XI. Ofrecer servicios adecuados a las necesidades locales y regionales;

Objetivo XII. Desarrollar funciones de vinculación con los sectores público, privado y social, para contribuir al desarrollo económico y social de la comunidad" (Gobierno del Estado de Quintana Roo, 2006, p. 5)

Las actividades de vinculación pueden darse en las siguientes formas: a) a través de cursos abiertos dirigidos a la comunidad; b) mediante proyectos de investigación y/o de solución de problemáticas de las comunidades o de la región; c) con actividades culturales; d) por medio de la organización de talleres, congresos, seminarios, etc., ya sea dirigidos a alguna organización o institución académica en particular o abierto al público en general; e) a través de publicaciones de folletos, noticias en los medios de comunicación, libros, etc.; f) con las actividades de los veranos que desarrollan los estudiantes con la supervisión y asesoría de los profesores (UIMQROO, 2006).

El servicio social en la UIMQROO se cubre a partir de trabajo de campo. Se concreta a través de Talleres de Vinculación con la Comunidad que se realizan durante el período de vacaciones de verano durante los cuatro años que dura el programa de licenciatura; de este modo se logra cubrir el total de horas necesarias.

A nivel normativo, el PEDI 2007-2017, dentro de las estrategias sobre docencia, inciso k, marca:

"El trabajo de campo permitirá: reencontrarse con su cultura y su lengua, reconocer su entorno, revalorar el potencial que tienen, confrontar la teoría con la práctica, identificar áreas de oportunidad para el desarrollo de sus comunidades, generar proyectos que puedan gestionarse para financiamiento". (UIMQROO, 2006, p. 43).

Dentro del campo organizativo, cada licenciatura coordina su propio programa de vinculación con la comunidad, además de que ciertas acciones se organizan de manera particular. Una de las actividades es Tianguis Agroecológico y Cultural, que se lleva a cabo un fin de semana de cada mes. En este caso, se organiza en coordinación con los departamentos, 
el municipio de José María Morelos y las asociaciones de productores locales de los municipios cercanos a la sede de la UIMQROO.

Con respecto al servicio social, debido al modelo educativo en donde los jóvenes trabajan en sus comunidades, se presentan algunas desventajas, entre ellas, que en ocasiones se pierden oportunidades de becas estudiantiles específicamente otorgadas para este propósito. Sin embargo, la UIMQROO busca la manera de compaginar los horarios de clases de los jóvenes para que estas ofertas puedan ser aprovechadas y los estudiantes tengan otra experiencia laboral además de las prácticas comunitarias (UIMQROO, 2006, p. 45).

El campo normativo se enlaza con el organizativo desde el modelo educativo de la UIMQROO, donde se planeó la creación de diversos centros que brindaran un enlace con la comunidad y los resultados de las investigaciones se relacionaran de modo más expedito con las demandas del entorno (UIMQROO, 2006, p. 50).

Actualmente, la Universidad cuenta con tres centros de investigación que dan respuesta a los planteamientos anteriores: el Centro Intercultural de Proyectos y Negocios, el Centro Intercultural de Certificación y Evaluación, y el Centro de Innovación para el Desarrollo Apícola Sustentable. De modo adicional, con respecto a las diferentes ramificaciones de la vinculación con el sector productivo, promueve las prácticas profesionales de sus estudiantes y presta sus instalaciones a distintos actores sociales.

UTRM $^{9}$

La Universidad Tecnológica de la Riviera Maya se localiza en la ciudad de Playa del Carmen, en la zona norte de Quintana Roo. Su fundación data del año 2005 y pertenece al subsistema de Universidades Tecnológicas. Ofrece seis programas para Técnico Superior Universitario (TSU), dos licenciaturas y tres ingenierías. Su matrícula era de 1269 alumnos en el año 2016. El centro educativo se encuentra en las afueras de la ciudad de Playa del Carmen, dentro de una zona que actualmente se está urbanizando con una enorme rapidez.

En sus orígenes, la UTRM impartía solo programas educativos para TSU, y hoy cuenta con seis de ellos: Tecnologías de la Información y Comunicación, Turismo, Gastronomía, Terapia Física, Mantenimiento y Administración. También imparte tres ingenierías: Mantenimiento Industrial, Tecnologías de la Información, Desarrollo e Innovación Empresarial; y dos licenciaturas: Gestión y Desarrollo Turístico, Gastronomía (UTRM, 2017).

Señala que es su misión:

"Brindar servicios educativos con programas académicos enfocados a las necesidades del sector productivo, sustentados en una infraestructura tecnológica y de alta calidad académica que haga posible el desarrollo de conocimientos y habilidades de nuestros educandos con un sentido de equidad e igualdad social". (UTRM, 2017).

Es importante mencionar que no cuenta en su organigrama con una instancia institucional dedicada a la extensión universitaria sino solamente con la denominada Dirección de Vinculación, de la cual depende el Departamento de Prácticas y Estadías, que es la instancia que lleva cabo con más claridad la relación de la UTRM con su entorno social. 
Como mecanismo concreto de enlace con el entorno, aparece:

"Artículo 44. El servicio social comunitario fortalece la colaboración de las instituciones de educación superior con los diversos sectores de la sociedad al contribuir a la solución de problemas locales, tales como la baja productividad, la higiene, el cuidado del medio ambiente y otros requerimientos sociales". (Honorable Poder Legislativo, 2005, p. 27)

A nivel operativo, de acuerdo con el Reglamento Académico y de Becas, las estadías profesionales son equivalentes al servicio social (Consejo Directivo, 2015, p. 28), por lo cual las estrategias que desarrolla la Dirección de Vinculación utilizan otros esquemas. Uno de ellas es la integración a la Brigada Ambiental, opción de "Jaguares. Cultura y Deporte", que involucra cubrir determinado número de horas como requisito de titulación; a los estudiantes les llegan invitaciones del ayuntamiento y del Estado para limpieza de playas o tutorías de alumnos de secundaria con problemas académicos. Otros ejemplos serían el "Paseo en bicicleta por el autismo"; K'Ame'Ex Kiimak Óolaal, segunda feria de intercambio de semillas nativas; Kiwik, turismo sostenible para el empoderamiento de las comunidades y emprendedores sociales; los tres se realizaron en 2017.

Por su parte, la vinculación con el sector productivo es muy relevante, puesto que se plantea que se debe:

"Planear, formular, desarrollar y operar programas y acciones de investigación tecnológica y servicios tecnológicos, prestar servicios de asesoría, apoyo administrativo y técnico, capacitación técnica, elaboración y desarrollo de proyectos de ingeniería, supervisión, estudios y actividades en materia de seguridad, salud y medio ambiente, estudios y desarrollo de proyectos geológicos, exploración, explotación y producción de hidrocarburos y demás áreas del sector energético y servicios diversos al sector público, social y privado. (Poder Ejecutivo de Quintana Roo, 2015, p. 20)

Por ello, en el Consejo Directivo, órgano de mayor autoridad de la institución, participan tres representantes del sector productivo. Se busca que mediante la vinculación se posicione a la institución y se facilite a los egresados su aceptación en el sector productivo de la zona.

En cuanto a la vertiente de profesionalización de los estudiantes, el modelo educativo de la UTRM establece como uno de sus tres pilares el "Eje Escuela-Planta Productiva. La enseñanza-aprendizaje debe estar plenamente compenetrada en el ámbito empresarial, por lo que este eje determina una estadía de 10 a 15 semanas que se cursa en las empresas durante el sexto cuatrimestre (UTRM, 2003). Los estudiantes deben tener una "estadía en el sector productivo", por lo tanto, las prácticas profesionales cuentan con un asesor académico nombrado por la UTRM y un asesor empresarial asignado por la organización pública o privada en la cual se esté desempeñando (Consejo Directivo, 2015, p. 2). Desde su fundación al año 2017 se firmaron alrededor de 290 convenios con hoteles y otros ramos del sector turístico, aunque se reconocen algunas desventajas, pues en ocasiones las empresas buscan tener la mano de obra barata, sin pagar las prestaciones correspondientes.

Como podemos ver, las IES de la Riviera Maya tienen una fuerte direccionalidad hacia el sector económico del turismo. La zona en que se ha desarrollado cumple con las características propias de la atracción del gran turismo internacional. Si bien está asentada en una zona del Estado de Quintana Roo muy cercana a la población indígena maya, es fundamental 
recordar que es zona hotelera a todo lo largo de su costa y sus asentamientos humanos más importantes, Cancún, Playa del Carmen, Cozumel, son desarrollos urbanos de muy reciente crecimiento asociado al turismo. Donde no había habitante alguno o un pequeño poblado hace unas décadas, ahora es un centro muy atractivo para el turismo internacional. Cabe mencionar que la propia denominación de Riviera Maya es el nombramiento reciente que se ha hecho a esta zona para vestirla de un carácter turístico, pues combina las ideas del mar y la playa y de singularidad indígena de los pobladores mayas.

En este sentido, las IES han jugado un doble papel: por una parte, su creación está ligada a este desarrollo turístico, por lo cual se han convertido en formadoras de profesionistas dirigidos a esta industria, sobre todo en sus programas de estudio, donde se ofrecen las carreras de hotelería, turismo, gastronomía, etc., de tal manera que incluso han retomado en algunos casos la nomenclatura de esta industria incluyendo en su nombre la referencia a la denominación de carácter publicitario de la Riviera Maya. Por otra parte, han intentado plantear una serie de programas tanto en la docencia como en la investigación y, por supuesto, aunque en menor medida, en la extensión universitaria y la difusión cultural, orientados a lograr un desarrollo sostenible que se haga responsable del cuidado del medio ambiente y con una preocupación ecologista de protección de la zona habitada. Sin embargo, es claro que al final, cuando se habla de cuidado ambiental, precisamente por las características que hemos enunciado de su perfil económico como zona turística internacional, su tendencia está claramente dirigida hacia una lógica de trato hacia el medio ambiente como una parte del desarrollo económico (Bustillo y Martínez, 2008). Aun así, los programas de extensión universitaria encontrados poseen estructuras sólidas y han mostrado tener continuidad, lo cual resulta en el fortalecimiento de su participación en los temas del medio ambiente de la zona.

Por supuesto que este tipo de desarrollo, sobre todo en lo que respecta a la responsabilidad universitaria para con el medio ambiente, hace que sean muy valiosos los esfuerzos que a través de los programas de extensión universitaria se realizan con un doble objetivo: dirigir sus programas al beneficio social y económico para lograr una sociedad menos desigual e intentar tener programas de desarrollo que atiendan a planteamientos de cuidado del ambiente y a la protección del equilibrio ecológico.

\section{Conclusiones}

En este artículo hemos querido mostrar que en el Sistema de Educación Superior Pública en México, en su diversidad y complejidad, las modalidades de extensión universitaria y difusión cultural responden a ello y a su especificidad territorialidad.

Las dos regiones que presentamos tienen una ubicación geográfica relativamente cercana y una fuerte composición de población indígena. Una, la región de Chiapas, de manera más tradicional y comunitaria, y la otra, la de la región de la Riviera Maya, inserta en una lógica de desarrollo económico de carácter modernizante. La primera, con una fuerte marginación económica y con índices de pobreza por demás altos, plantea reivindicaciones sociales y políticas de justicia social con la presencia relevante del EZLN, que en el transcurso de las últimas décadas ha dado cauce a la voz de indignación de los pobladores de la zona; la segunda, ligada desde su misma génesis a una industria hotelera basada en el desarrollo comercial y económico. 
En ambas regiones, las IES intervienen para llevar a cabo programas de docencia, en su caso, de investigación y de extensión universitaria, que buscan solventar las distintas problemáticas que las afectan con una idea de cuidado ambiental y equilibrio ecológico matizada en cada una por el carácter del desarrollo económico y social que la ha marcado. Como hemos dicho, en la zona indígena de Chiapas, la Universidad está más ligada a la comunidad de manera crítica, aunque, también hay que decirlo, resulta tan compleja política y socialmente que poco permite una inserción clara en el proceso de desarrollo de la región y del país. En tanto, en la zona turística de la Riviera Maya se coloca el foco en el mercado de trabajo que está encauzado al gran turismo internacional.

Con ello, resulta relevante poder esbozar nuevamente nuestra tesis central, a saber: que a pesar de que las IES estudiadas en ambas regiones son parte de un mismo Sistema de Educación Superior Pública que presenta características institucionales semejantes, la determinación de su territorialidad define de manera relevante la intencionalidad y los objetivos concretos de las acciones específicas que llevan a cabo en cada región. A pesar de ser el mismo Sistema, con los mismos tipos de instituciones públicas, y tener, dad esta direccionalidad institucional, objetivos semejantes, cada región, entendida como el contexto económico, social y cultural, acaba por darle un carácter distintivo en lo referente, entre otros temas, a la cuestión ambiental y al cuidado de la naturaleza. En este sentido, la extensión universitaria de las IES no tiene un sentido unívoco sino que es un verdadero crisol de experiencias.

\section{Referencias bibliográficas}

Arzeno, M. (2018). Extensión en el territorio y territorio en la extensión. Aportes a la discusión desde el campo de la Geografía. +E: Revista de Extensión Universitaria, 8(8), 3-11. 10.14409/extensión.v8i8.Ene-Jun.7709

Barradas, S.; Ejea, T. y Molina, A. (2017). Reporte de estudio de caso Región Chiapas. Mimeo. Universidad Veracruzana. https://www.academia.edu/49444417/Reporte_de_investigacion_chiapas

Bohne, A.; Bruckmann, M.; y Martínez, A. (2019). El desarrollo sustentable en las instituciones de educación superior: un verdadero desafío. Revista Digital Universitaria, 20(5). UNAM. México. https://www.revista.unam. mx/2019v20n5/el-desarrollo-sustentable-en-las-instituciones-de-educacion-superior-un-verdadero-desafio/ Bustillo, L. y Martínez, J. P. (2008). Los enfoques del desarrollo sustentable. Interciencia, 33(5). INCI. Caracas. CGEIB (Coordinación General de Educación Intercultural y Bilingüe) (2015). Matrícula total de las Universidades Interculturales. Dirección de Educación Media Superior y Superior (Ciclo Escolar 2015-2016). http://eib. sep.gob.mx/diversidad/wp-content/uploads/2016/06/Matriculatotal-15-16-Octubre-2015.pdf

Chiu, S. (2017). Universidad Intercultural Maya de Quintana Roo. En: Sainz, I.; Ejea, T. y Molina, A. (2017). Reporte de estudio de caso Región Riviera Maya. Mimeo. Universidad Veracruzana. https://www.academia. edu/49444911/Reporte_de_investigacion_riviera_maya

Consejo Directivo de la Universidad Tecnológica de la Riviera Maya (2015). Reglamento Académico y de Becas de la Universidad Tecnológica de la Riviera Maya. Playa del Carmen. http://www.utrivieramaya.edu.mx/ utrm1/marco-juridico/reglamentos-vigentes

De Vries, W. y Rivera, C. (2017). La educación superior mexicana y el desarrollo sustentable. En Políticas públicas para el Desarrollo Sustentable: Horizonte en el Siglo XXI. BUAP.

Erreguerena, F. (2020). Repolitizar los territorios. Reflexiones sobre los conceptos de territorio y poder en la extensión universitaria. +E: Revista de Extensión Universitaria, 10(13), e0012. 10.14409/extension.2020.13. Jul-Dic.e0012 
Gasca, J. (2009). Geografía regional. La región, la regionalización y el desarrollo regional en México. UNAM. Gobierno del Estado de Quintana Roo (2006). Decreto de Creación de la UIMQROO. Periódico Oficial del Gobierno del Estado de Quintana Roo (p. 16). http://www.uimqroo.edu.mx/Documentos/NuestraUniversidad/ decreto-30-10-06.pdf

Harvey, N. (2003). La rebelión en Chiapas: la lucha por la tierra y la democracia. ERA.

Honorable Poder Legislativo (2005). Ley de Educación del Estado de Quintana Roo. Periódico Oficial del Estado de Quintana Roo (p. 45). Chetumal. http://www.uqroo.mx/nuestra-universidad/documentos/\%0A

INEGI (Instituto Nacional de Estadística y Geografía) (2016a). México en cifras: Chiapas. http://www.beta.inegi. org.mx/app/areasgeograficas/?ag=07 (el 16/08/2017)

(2016b). Conociendo Chiapas. 5ta. ed. INEGI.

(2016c). Información por entidad, Quintana Roo. http://cuentame.inegi.org.mx/monografias/informacion/pue/poblacion/ePNUD 2014

García Villa, A. (1992). La Planificación de Centros Turísticos de México. Limusa.

Gobierno del Estado de Chiapas (2013). Plan Estatal de Desarrol/o 2013-2018. http://www.chiapas.gob.mx/ plan-estatal

Molina, A.; Barradas, S. y Torres, B. (2017). Universidad Intercultural de Chiapas. En Barradas, S.; Ejea, T. y Molina, A. (2017). Reporte de estudio de caso Región Chiapas. Mimeo. Universidad Veracruzana. https://www. academia.edu/49444417/Reporte_de_investigacion_chiapas

Molina, A. y Ejea, T. (2019). Entre la Comunidad y el Mercado. Los ámbitos y usos de la tercera función sustantiva en México. Universidad Veracruzana. https://www.academia.edu/40781895/Entre_la_comunidad_y_el_mercado_Los_\%C3\%A1mbitos_y_usos_de_la_tercera_funci\%C3\%B3n_sustantiva_universitaria_en_M\%C3\%A9xico Moneva, J. y Vallespín, E. (2012). Universidad y desarrollo sostenible: análisis de la rendición de cuentas de las universidades públicas desde un enfoque de responsabilidad social. Revista Iberoamericana de Contabilidad de Gestión, 10(19). España.

Pearce, D. (1991). Desarrollo Turístico, Su planeación y Ubicación Geográfica. Trillas.

PNUD (Programa de las Naciones Unidas para el Desarrollo) (2019). Informe sobre Desarrollo Humano en México 2019. Desigualdad y Movilidad. http://www.mx.undp.org/content/dam/mexico/docs/Publicaciones/PublicacionesReduccionPobreza/InformesDesarrolloHumano/idhmovilidadsocial2019/PNUD\%20IDH2016.pdf

Poder Ejecutivo de Chiapas, Secretaría de Gobierno (2004). Decreto de Creación. Universidad Intercultural de Chiapas. Periódico Oficial, Tuxtla Gutiérrez, Chiapas, México. http://www.unich.edu.mx/wp-content/ uploads/2014/04/DECRETO-DE-CREACION-2014.pdf

Poder Ejecutivo de Quintana Roo (2015). Decreto por el que se Reforma Integralmente el Decreto por el que se Crea la Universidad Tecnológica de la Riviera Maya. Periódico Oficial del Estado de Quintana Roo. Chetumal. http://transparencia.utrivieramaya.edu.mx/wp-content/uploads/2017/02/Decreto-de-laUniversidad-Tecnológica-de-la-Riviera-Maya-reformado.pdf

Poder Judicial de Chiapas (2014). Ley de Educación para el Estado de Chiapas. Periódico Oficial del Estado de Chiapas. http://www.poderjudicialchiapas.gob.mx/forms/archivos/a5ecley-de-educacion-para-el-estado-de-chiapas.pdf

Sainz, I. y Torres, B. (2017). Universidad Tecnológica de la Riviera Maya. En Sainz, I.; Ejea, T. y Molina, A. (2017). Reporte de estudio de caso Región Riviera Maya. Mimeo. Universidad Veracruzana. https://www.academia.edu/49444911/Reporte_de_investigacion_riviera_maya

Secretaría de Educación Pública (2017). Secretaría de Educación Pública. Obtenido de Subsecretaría de Educación Superior. http://www.ses.sep.gob.mx/interculturales.html

Secretaría de Gobierno de Chiapas (1997). Decreto por el que se crea la Universidad Tecnológica de la Selva. 
Periódico Oficial del Estado de Chiapas. http://www.utselva.edu.mx/MarcoLegal/MarcoLegal/Decreto/Decreto-UTSelva-Periodico_-Oficial-No57-19-Nov-1997.pdf

Schmelkes, S. (2008). Las Universidades Interculturales en México: ¿Una contribución a la equidad en educación superior? Universidad Iberoamericana.

Tommasino, H. y Cano, A. (2016). Modelos de extensión universitaria en las universidades latinoamericanas en el siglo XXI: tendencias y controversias. Universidades, (67). México.

Torres, B. (2017). Universidad Tecnológica de la Selva. En Barradas, S.; Ejea, T. y Molina, A. (2017). Reporte de estudio de caso Región Chiapas. Mimeo. Universidad Veracruzana. https://www.academia.edu/49444417/ Reporte_de_investigacion_chiapas

UNICH (Universidad Intercultural de Chiapas) (2013). Plan Institucional de Desarrollo 2013-2024. https://www. unich.edu.mx/wp-content/uploads/2014/03/PIDE-2013-2024.pdf

UIMQROO (Universidad Intercultural Maya de Quintana Roo) (2006). Plan Estratégico de Desarrollo Institucional 2007-2017. https://www.uimqroo.edu.mx/Documentos/PEDI/PEDI\%20UIMQRoo\%202007-2017.pdf

UTRM (Universidad Tecnológica de la Riviera Maya) (2003). Modelo educativo. http://www.utrivieramaya.edu. mx/utrm1/modelo-educativo

(2017). Universidad Tecnológica de la Riviera Maya. http://www.utrivieramaya.edu.mx/

UT de la Selva (Universidad Tecnológica de la Selva) (2013). Programa Institucional Universidad Tecnológica de la Se/va 2013-2018. http://www.utselva.edu.mx/contacto/transparencia/Documento-Plan-UTSelva-Actual.pdf (2016). Informe del Rector 2016.

Yánez, R. y Zavarce, C. (2011). Desarrollo sustentable, universidad y gestión del conocimiento desde la perspectiva luhmaniana. Revista Iberoamericana de Ciencia, Tecnología y Sociedad, 6(17). Centro de Estudios sobre Ciencia, Desarrollo y Educación Superior. Buenos Aires. 